\title{
The Association of ADRA2A Genotype with Dexmedetomidine Dosage in Pediatric Patients
}

\author{
Ashwin Biju1, Katherine A. Gallaway ${ }^{1}$, Todd C. Skaar1, Emma M. Tillman¹
}

1Division of Clinical Pharmacology, Indiana University School of Medicine, Indianapolis Indiana

\section{Background:}

Critically ill children in the pediatric intensive care unit (PICU) often require sedation with dexmedetomidine, an $\alpha_{2}$-adrenergic receptor $(A D R A 2 A)$ agonist. In adults, evidence suggests an ADRA2A polymorphism (rs1800544) modulates the dexmedetomidine response. We hypothesize that pediatric patients with at least one wild-type allele (GG and GC) will require larger dexmedetomidine doses and a longer time to effective dose than patients with a variant genotype (CC).

\section{Methods:}

This study was approved by the institutional review board (IRB). Patients in the PICU, 1 week through 25 years of age, and sedated with dexmedetomidine for $\geq 2$ days were approached for enrollment. Genomic DNA was extracted from blood or saliva before genotyping for rs1800544 with a custom designed TaqMan ${ }$ Assay on the QuantStudio 12K Flex platform. Patient genotype was determined using TaqMan® Genotyper software. Patient data were collected from the electronic medical record.

\section{Results:}

Sixty-five patients were enrolled and genotyped. Twenty-six patients were homozygous variant (CC), 27 heterozygous (GC), and 12 wildtype (GG) for rs 1800544 . The maximum dose $(\mathrm{mcg} / \mathrm{kg} / \mathrm{hr})$ was $0.75 \pm 0.31$, and $0.78 \pm 0.32$, in patients with and without a $\mathrm{G}$ allele, respectively $(p=0.65)$. The mean doses were $0.59 \pm 0.22$ and $0.58 \pm 0.24$ in patients with and without a $G$ allele, respectively $(p=0.85)$. The time to effective dose (hrs) was $5.8 \pm 12.0$ for patients with a $G$ allele and $4.0 \pm 8.2$ for patients with no $G$ allele $(p=0.74)$.

\section{Conclusion:}

Our hypothesis that the CC genotype would require lower doses of dexmedetomidine was not confirmed. Patients without a $\mathrm{G}$ allele required lower dexmedetomidine dosage and a longer time to achieve an effective dose, but these findings were not significant. This could be due to the small sample size or clinical factors that affect pediatric sedation. An adequately powered study could determine the association of ADRA2A genotype with dexmedetomidine. 